neben der Zulassung als Voraussetzung für die Verordnungsfähigkeit zulasten der gesetzlichen Krankenversicherung (GKV) auch die einschlägigen Bestimmungen der Arzneimittel-Richtlinien des Gemeinsamen Bundesausschusses (G-BA) zu beachten sind. Bei verschreibungspflichtigen Arzneimitteln sind dort Ausschlüsse definiert, die sowohl bei Erwachsenen wie auch bei Kindern ohne Einschränkung gelten. Ausnahmsweise ist in medizinisch begründeten Fällen eine Verordnung trotzdem möglich ( $\$ 31$ Abs. 1 Satz 4 $S G B V$ ). Nicht verschreibungspflichtige Arzneimittel sind hingegen grundsätzlich von der Verordnung ausgeschlossen, ausgenommen bei Kindern bis zum 12. Lebensjahr und Jugendlichen mit Entwicklungsstörungen bis zum 18. Lebensjahr. Darüber hinaus hat der G-BA im Einzelfall festgelegt, welche dieser Arzneimittel bei welchen Indikationen ausnahmsweise doch verordnungsfähig sind.

\title{
Rentenversicherungsgutachten nicht zum Dumpingpreis
}

\begin{abstract}
— Da seit dem 1. Juli 1997 keine Honorarvereinbarung mehr zwischen Bundesärztekammer und Deutscher Rentenversicherung besteht, ist für die Ärzteschaft eine außerordentlich unbefriedigende Situation hinsichtlich der Vergütung von Befundberichten und Gutachten im Auftrag der Deutschen Rentenversicherung entstan-

den. Zurzeit finden zwar Gespräche zwischen Bundesärztekammer und der Deutschen Rentenversicherung Bund statt, in denen die Möglichkeiten einer neuen bundeseinheitlichen Honorierungsregelung für Befundberichte erörtert werden. Ob eine Einigung gelingen wird, ist zum jetzigen Zeitpunkt allerdings völlig unklar.
\end{abstract}

\section{Tabelle 1}

\section{Nach diesen Sätzen des Justizvergütungs- und Entschädigungs- gesetzes (JVEG) könnten Rentengutachten liquidiert werden}

\section{Gebühr}

200

201

202

203
Leistungsbeschreibung

Ausstellung eines Befundscheines oder Erteilung einer schriftlichen Auskunft ohne nähere gutachtliche Äußerung.

Die Leistung der in Nr. 200 genannten Art ist außergewöhnlich umfangreich. ranziehenden Stelle geforderter kurzer gutachtlicher Äußerung oder Formbogengutachten, wenn sich die Fragen auf Vorgeschichte, Angaben und Befund beschränken und nur ein kurzes Gutachten erfordern.

Die Leistung der in Nr. 202 genannten Art ist außergewöhnlich umfangreich. bis zu

44,00

38,00

\section{Euro}

21,00

38,00

\section{MMW Kommentar}

Interessant ist in diesem Zusammenhang ein Hinweis der Ärztekammer Westfalen-Lippe. Da das Sozialgesetzbuch eine Auskunftspflicht der behandelnden Ärzte gegenüber dem Träger der Rentenversicherung vorsieht ( $\$ 100$ SGB X), kann man nach deren Auffassung eine Bearbeitung der Anfragen der Deutschen Rentenversicherung und deren Arbeitsgemeinschaften zwar nicht verweigern. Die Träger der Rentenversicherung könnten aber aufgefordert werden, Gutachten bzw. Befundberichte nicht zu der angebotenen Entschädigung, sondern nach dem Justizvergütungs- und Entschädigungsgesetz (JVEG) zu vergüten.

Diese Vorgehensweise ist sogar durch ein Urteil des Sozialgerichts Detmold abgesichert. Nach Auffassung der Richter sehen die Verträge mit den Rentenversicherungsträgern weder ausdrückliche noch konkludente Erklärungen vor, die eine Einigung hinsichtlich einer bestimmten Höhe der Entschädigung für die Erstattung des Befundberichtes ausschließen (Az: S9 (7) RJ 46/98).

\section{Hautkrebsscreening: wie oft berechnungsfähig?}

- Das Hautkrebsscreening (HKS) kann nach den Krebsfrüherkennungs-Richtlinien ab einem Alter des Versicherten von 35 Jahren jedes zweite Jahr berechnet werden. Viele Kassen haben mit den regionalen KVen auch weitergehende Verträge geschlossen, die eine Berechnung ab 18
Jahren oder ohne Altersbeschränkung vorsehen. Bei anderen Regelungen bleibt es bei der Anspruchsberechtigung von 35 Jahren, das Screening ist jedoch jährlich berechnungsfähig. In vielen Fällen sind dann auch gesonderte Abrechnungsziffern in der Abrechnung anzugeben.

\section{MMW Kommentar}

Die erwähnten Sonderregelungen gelten. In Ausnahmefällen ist aber auch eine Leistungserbringung und Abrechnung durch Hausärzte zulässig. 